\title{
VARIABLE EXPONENT SOBOLEV SPACES ON METRIC MEASURE SPACES
}

Petteri Harjulehto* ${ }^{*}$ Peter Hästö** and Mikko Pere

Abstract: In this article we study variable exponent Sobolev spaces on metric measure spaces. We employ two definitions: a Hajłasz type definition, which uses a pointwise maximal inequality, and a Newtonian type definition, which uses an upper gradient. We prove that these spaces are Banach, that Lipschitz functions are dense as well as other basic properties. We also study when these spaces coincide.

Keywords: Variable exponent, Sobolev space, metric measure space, Newtonian space, Hajłasz space.

\section{Introduction}

The theory of Sobolev spaces was originally developed in domains of $\mathbb{R}^{n}$ using the notion of distributional derivatives. To generalize this theory to metric spaces alternative ways to define Sobolev spaces were needed. P. Hajłasz showed in [12] that a $p$-integrable function $u, 1<p<\infty$, belongs to $W^{1, p}\left(\mathbb{R}^{n}\right)$ if and only if there exists a non-negative $p$-integrable function $g$ such that

$$
|u(x)-u(y)| \leqslant|x-y|(g(x)+g(y))
$$

for almost every $x, y \in \mathbb{R}^{n}$. This inequality can be stated also in metric measure spaces if $|x-y|$ is replaced by the distance between the points $x$ and $y$. Spaces defined using this inequality are often called Hajłasz spaces.

Another way to define Sobolev spaces on metric measure spaces is via the concept of an upper gradient. A non-negative function $\rho$ is said to be an upper gradient of $u$ if

$$
|u(x)-u(y)| \leqslant \int_{\gamma} \rho d s
$$

2000 Mathematics Subject Classification: 46E35.

* Supported in part by the Academy of Finland.

** Supported in part by a Gehring-Finland Postdoctoral Fellowship and by the Finnish Academy of Science and Letters. 
for every $x, y$ and curve $\gamma$ connecting $x$ to $y$. In an open set $\Omega$ of $\mathbb{R}^{n}$ this holds for a Sobolev function $u$ on every curve not belonging to an exceptional family of $p$-modulus zero [11]. If this holds in a general metric space, we call $g$ a weak upper gradient of $u$. Existence and $p$-integrability of a weak upper gradient together with $p$-integrability of the function lead to another characterization of $W^{1, p}(\Omega)$. These spaces are often called Newtonian spaces. For basic properties of Newtonian spaces see the pioneering work of N. Shanmugalingam [29].

Sobolev spaces on metric measure spaces have been studied very intensively during the last ten years, and the "standard" framework is now available in the monograph [18] by J. Heinonen. On the other hand new approaches are still being put fourth, see e.g., [13]. For instance it is known that if the measure is doubling and the space supports a $(1, p)$-Poincaré inequality, then the Hajłasz and Newtonian spaces coincide. These advances have made possible the development of potential theory in metric measure spaces, see for example $[1,19]$.

Variable exponent Lebesgue and Sobolev spaces have attracted a steadily increasing interest over the last couple of years, but most papers have dealt only with the Euclidean case. Variable exponent spaces have been independently discovered by several investigators $[9,21,28,30]$ and are related to differential equations with non-standard coercivity conditions. For some of the latest advances in the Euclidean theory see $[6,15,20]$.

In metric measure spaces the variable exponent is very natural. For example, it allows us to study Sobolev spaces with integrability connected to the dimension of the space, which changes with location. Only three papers exist on variable exponent spaces on metric measure spaces, two by T. Futamura, Y. Mizuta and T. Shimomura [10, 23] and one by the authors [17]. All of these papers deal only with variable exponent Lebesgue spaces.

In this article we study Hajłasz and Newtonian spaces with variable exponent in metric measure spaces. In the next section we review some definitions and present the theory of variable exponent Lebesgue spaces on metric measure spaces. In Section 3 we show that the variable exponent Newtonian space is a Banach space. We prove that Lipschitz continuous functions are dense if the measure is doubling and the space supports a Poincaré inequality. In the final section we study when Hajłasz and Newtonian spaces coincide in a metric measure space and also in Euclidean space.

\section{Preliminaries}

This section contains some material on variable exponent Lebesgue spaces. The results in the first two sections are used throughout this paper. The last two sections are necessary only for specific parts, and can be read later as needed.

2.1. Metric measure spaces. By a metric measure space we mean a triple $(X, d, \mu)$, where $X$ is a set, $d$ is a metric on $X$ and $\mu$ is a non-negative Borel regular outer measure on $X$ which is finite in every bounded set. For simplicity, 
we often write $X$ instead of $(X, d, \mu)$. For $x \in X$ and $r>0$ we denote by $B(x, r)$ the open ball centered at $x$ with radius $r$. We use the convention that $C$ denotes a constant whose value can change even between different occurrences in a chain of inequalities.

A metric measure space $X$ or a measure $\mu$ is said to be doubling if there is a constant $C \geqslant 1$ such that

$$
\mu(B(x, 2 r)) \leqslant C \mu(B(x, r))
$$

for every open ball $B(x, r) \subset X$. The constant $C$ in (2.1) is called the doubling constant of $\mu$. By the doubling property, if $B(y, R)$ is an open ball in $X, x \in$ $B(y, R)$ and $0<r \leqslant R<\infty$, then

$$
\frac{\mu(B(x, r))}{\mu(B(y, R))} \geqslant C_{Q}\left(\frac{r}{R}\right)^{Q}
$$

for some $C_{Q}$ and $Q$ depending only on the doubling constant. For example, in $\mathbb{R}^{n}$ with the Lebesgue measure (2.2) holds with $Q$ equal to the dimension $n$.

We say that the measure $\mu$ is lower Ahlfors $Q$-regular if there exists a constant $C>0$ such that $\mu(B) \geqslant C \operatorname{diam}(B)^{Q}$ for every ball $B \subset X$ with $\operatorname{diam} B \leqslant 2 \operatorname{diam} X$. We say that $\mu$ is upper Ahlfors $Q$-regular if there exists a constant $C>0$ such that $\mu(B) \leqslant C \operatorname{diam}(B)^{Q}$ for every ball $B \subset X$ with $\operatorname{diam} B \leqslant 2 \operatorname{diam} X$. The measure $\mu$ is Ahlfors $Q$-regular if it is upper and lower Ahlfors $Q$-regular, i.e. if $\mu(B) \approx \operatorname{diam}(B)^{Q}$ for every ball $B \subset X$ with $\operatorname{diam} B \leqslant$ $2 \operatorname{diam} X$. If $X$ is a bounded doubling metric measure space, so that $\mu(X)<\infty$ and $\operatorname{diam}(X)<\infty$, then it is lower Ahlfors $Q$-regular.

2.2. Variable exponent Lebesgue spaces. We call a measurable function $p: X \rightarrow[1, \infty)$ a variable exponent. For $A \subset X$ we define $p_{A}^{+}=$ess $\sup _{x \in A} p(x)$ and $p_{A}^{-}=e s s \inf _{x \in A} p(x)$; we further abbreviate $p^{+}=p_{X}^{+}$and $p^{-}=p_{X}^{-}$. For a $\mu$-measurable function $u: X \rightarrow \mathbb{R}$ we define the modular

$$
\varrho_{p(\cdot)}(u)=\int_{X}|u(y)|^{p(y)} d \mu(y)
$$

and the norm

$$
\|u\|_{p(\cdot)}=\inf \left\{\lambda>0: \varrho_{p(\cdot)}(u / \lambda) \leqslant 1\right\} .
$$

Sometimes we use the notation $\|u\|_{p(\cdot), X}$ when we want to emphasize in what metric space the norm is taken. The variable exponent Lebesgue space $L^{p(\cdot)}(X, d, \mu)$ consists of those $\mu$-measurable functions $u: X \rightarrow \mathbb{R}$ for which $\|u\|_{p(\cdot)}<\infty$. This is a special case of an Orlicz-Musielak space, cf. [24].

As in the Euclidean setting, we easily see that $\|\cdot\|_{p(\cdot)}$ is a norm. Also, if $\|f\|_{p(\cdot)} \leqslant 1$, then $\varrho_{p(\cdot)}(f) \leqslant\|f\|_{p(\cdot)}$. Moreover, if $p^{+}<\infty$, then $\varrho_{p(\cdot)}\left(f_{i}\right) \rightarrow 0$ if and only if $\left\|f_{i}\right\|_{p(\cdot)} \rightarrow 0$. If $p: X \rightarrow(1, \infty)$, then Hölder's inequality,

$$
\|f g\|_{1} \leqslant C\|f\|_{p(\cdot)}\|g\|_{p^{\prime}(\cdot)}
$$


holds also in variable exponent Lebesgue spaces, where $p^{\prime}$ is the pointwise Hölder conjugate of $p$, i.e. $p(x)+p^{\prime}(x)=p(x) p^{\prime}(x)$.

Variable exponent Lebesgue spaces on metric measure spaces have been studied in $[10,17,23]$. Some of the basic results are that $L^{p(\cdot)}(X)$ is a Banach space if $p^{+}<\infty[17$, Lemma 3.1] and that continuous functions with compact support are dense in $L^{p(\cdot)}(X)$ provided $X$ is a locally compact doubling space and $p^{+}<\infty$ $[17$, Theorem 3.3]. As in [21, Theorem 2.8] we can prove that

$$
\|u\|_{p(\cdot)} \leqslant(1+\mu(X))\|u\|_{q(\cdot)}
$$

if $\mu(X)<\infty$ and $p(x) \leqslant q(x)$.

Recall that a Banach space is said to be uniformly convex if for every $\delta>0$ there exists $\varepsilon>0$ such that $\|u\|=\|v\|=1$ and $\|u-v\|>\delta$ imply that $\|u+v\|<2-\varepsilon$. Recall also that a measure $\mu$ is atomless if $\mu(\{x\})=0$ for every point $x \in X$. For future reference we record the following simple but useful fact

Proposition 2.3. If $1<p^{-} \leqslant p^{+}<\infty$ and $\mu$ is atomless, then $L^{p(\cdot)}(X, \mu)$ is uniformly convex. From this it follows that $L^{p(\cdot)}(X, \mu)$ is reflexive and has the Banach-Saks property, namely, if $u_{i} \rightarrow u$ weakly, then $\frac{1}{i}\left(u_{1}+\ldots+u_{i}\right) \rightarrow u$ strongly.

The following condition has emerged as the right one to guarantee a high degree of regularity for variable exponent spaces in $\mathbb{R}^{n}$. We say that $p: X \rightarrow[1, \infty)$ is $\log$-Hölder continuous if

$$
|p(x)-p(y)| \leqslant \frac{C}{-\log d(x, y)},
$$

when $d(x, y) \leqslant 1 / 2$. This condition has also been called Dini-Lipschitz, weak-Lipschitz and 0-Hölder. Since it is the limiting case of $\alpha$-Hölder continuity, we think that $\log$-Hölder is the most descriptive term. The following lemma illustrates the geometrical significance of log-Hölder continuous exponents. It corresponds to Lemma 3.2 of [4] on the Euclidean case.

Lemma 2.5. [Lemma 3.6, [17]] Assume that $p^{+}<\infty$ and consider two conditions:

(i) $p$ is $\log$-Hölder continuous;

(ii) for all balls $B \subset X$ we have $\mu(B)^{p_{B}^{-}-p_{B}^{+}} \leqslant C$.

If $\mu$ is lower Ahlfors $Q$-regular, then (i) implies (ii). If $\mu$ is upper Ahlfors $Q$-regular, then (ii) implies (i).

2.3. The Hardy-Littlewood maximal operator. Recall that the Hardy-Littlewood maximal operator is defined for a locally integrable function $u$ by

$$
\mathcal{M} u(x)=\sup _{r>0} f_{B(x, r)}|u(y)| d \mu(y) .
$$


Recall also that the integral to the right denotes the mean value of $u$ in $B(x, r)$. Assume that $1<p^{-} \leqslant p^{+}<\infty, p$ is log-Hölder continuous and satisfies the decay estimate

$$
|p(x)-p(y)| \leqslant \frac{C}{\log (e+|x|)}
$$

for every $x, y \in \mathbb{R}^{n},|y| \geqslant|x|$. Then Cruz-Uribe, Fiorenza and Neugebauer proved that the Hardy-Littlewood maximal operator is bounded from $L^{p(\cdot)}\left(\mathbb{R}^{n}\right)$ to itself [3, Theorem 1.5]. In the local case this result was first derived by Diening [4]. Nekvinda [25] has given another global version of the boundedness result, using a decay condition stated in terms of an integral. Pick and Růžička [27] constructed an example which shows that log-Hölder continuity is in some sense sharp. Nekvinda has given an example which shows that the decay condition is not necessary [26], and Diening [5] has further studied the necessity of decay conditions.

In bounded doubling metric measure spaces the Hardy-Littlewood maximal operator is bounded if $p$ is log-Hölder and satisfies $1<p^{-} \leqslant p^{+}<\infty,[17$, Theorem 4.3]. However, in this case log-Hölder continuity is not necessary [17, Example 4.5]. A weaker result, derived under fewer assumptions, was given in [10, Theorem 2.3].

2.4. Sobolev spaces. Variable exponent Sobolev spaces are defined in the obvious way: For $\Omega \subset \mathbb{R}^{n}$ the variable exponent Sobolev space $W^{1, p(\cdot)}(\Omega)$ is the subspace of functions $u \in L^{p(\cdot)}(\Omega)$ whose distributional gradient exists almost everywhere and satisfies $|\nabla u| \in L^{p(\cdot)}(\Omega)$. The norm $\|u\|_{1, p(\cdot)}=\|u\|_{p(\cdot)}+\|\nabla u\|_{p(\cdot)}$ makes $W^{1, p(\cdot)}(\Omega)$ a Banach space.

For the Newtonian space to agree with the classical Sobolev space the density of differentiable functions turns out to be crucial. This question is not as well understood in variable exponent spaces as is the boundedness of the maximal operator, but we do have some results: Samko proved in [28] that smooth function are dense in $W^{1, p(\cdot)}\left(\mathbb{R}^{n}\right)$ if $p^{+}<\infty$ and $p$ is log-Hölder continuous. Diening proved a similar, though slightly weaker, result [4]. Edmunds and Rákosník showed that a certain monotonicity condition on the exponent is also sufficient for the density of smooth functions, see [8]. Hästö [12] gave an example of a variable exponent Sobolev space in which continuous functions are not dense. In this example the continuous exponent has growth just slightly greater than allowed by log-Hölder continuity.

\section{Newtonian spaces}

In this section we define Sobolev spaces on metric measure spaces using an upper gradient. These so-called Newtonian spaces spaces were first studied, in the fixed exponent case, by N. Shanmugalingam [29], see also [2].

A curve $\gamma$ in $X$ is a non-constant continuous map $\gamma: I \rightarrow X$, where $I=[a, b]$ is a closed interval in $\mathbb{R}$. The image of $\gamma, \gamma(I)$, is denoted by $|\gamma|$. By $\Gamma_{\text {rect }}$ we denote the family of all rectifiable curves in $X$. 
Let $\Gamma$ be a family of rectifiable curves. We denote by $F(\Gamma)$ the set of all admissible functions, i.e. all Borel measurable functions $\rho: X \rightarrow[0, \infty]$ such that

$$
\int_{\gamma} \rho d s \geqslant 1
$$

for every $\gamma \in \Gamma$, where $d s$ represents integration with respect to path length. We define the $p(\cdot)$-modulus of $\Gamma$ by

$$
\mathrm{M}_{p(\cdot)}(\Gamma)=\inf _{\rho \in F(\Gamma)} \int_{X} \rho(x)^{p(x)} d \mu(x) .
$$

If $F(\Gamma)=\emptyset$, then we set $\mathrm{M}_{p(\cdot)}(\Gamma)=\infty$. The arguments from $\mathbb{R}^{n}$ imply that the $p(\cdot)$-modulus is an outer measure on the space of all curves of $X$, for the proof see [16, Lemma 2.1].

A family of curves $\Gamma$ is said to be exceptional if $\mathrm{M}_{p(\cdot)}(\Gamma)=0$. The following lemma is a generalization of [11, Theorem $3(\mathrm{f})]$. The proof is exactly the same as the proof of [16, Lemma 2.2].

Lemma 3.1. [Fuglede's lemma] Let $\left(u_{i}\right)_{i=1}^{\infty}$ be a sequence of non-negative Borel functions in $L^{p(\cdot)}(X)$ converging to zero in $L^{p(\cdot)}(X)$. Then there exists a subsequence $\left(u_{i_{k}}\right)_{k=1}^{\infty}$ and an exceptional family $\Gamma$ of rectifiable curves such that for every rectifiable $\gamma \notin \Gamma$ we have

$$
\lim _{k \rightarrow \infty} \int_{\gamma} u_{i_{k}} d s=0 .
$$

3.1. Basic properties. Let $u$ be a real valued function on $X$. A non-negative Borel measurable function $\rho$ on $X$ is a $p(\cdot)$-weak upper gradient of $u$, or weak upper gradient for short, if there exists a family $\Gamma$ of rectifiable curves with $\mathrm{M}_{p(\cdot)}(\Gamma)=0$ and

$$
|u(x)-u(y)| \leqslant \int_{\gamma} \rho d s
$$

for every rectifiable curve $\gamma \notin \Gamma$ with endpoints $x$ and $y$.

The Newtonian space $N^{1, p(\cdot)}(X)$ is the collection of functions in $L^{p(\cdot)}(X)$ with a weak upper gradient in $L^{p(\cdot)}(X)$ equipped with the norm

$$
\|u\|_{N^{1, p(\cdot)}(X)}=\|u\|_{p(\cdot)}+\inf \|\rho\|_{p(\cdot)},
$$

where the infimum is taken over all weak upper gradients of $u$. It is easy to see that $N^{1, p(\cdot)}(X)$ is a lattice like classical first order Sobolev space.

Next we show that Newtonian space is a Banach space. For that purpose we introduce a Sobolev type capacity in Newtonian space and study the relation between the capacity and the modulus. Our proof is along the lines of the proofs of [16] and is shorter than the original proof of Shanmugalingam [29]. 
We define the capacity in Newtonian space by

$$
c_{p(\cdot)}(E)=\inf _{u} \inf _{\rho} \int_{X}|u(x)|^{p(x)}+\rho(x)^{p(x)} d \mu(x)
$$

where the first infimum is taken over all $u \in N^{1, p(\cdot)}(X)$, which are at least 1 in $E$ and the second infimum is taken over weak upper gradients of $u$. If the class of test functions is empty we set $c_{p(\cdot)}(E)=\infty$. Standard arguments show that the capacity is an outer measure provided that $p^{+}<\infty$.

We denote by $\Gamma_{E}$ the family of all rectifiable curves whose image intersects the set $E$.

Lemma 3.2. Suppose that $E \subset X$ and $p^{+}<\infty$. If $c_{p(\cdot)}(E)=0$, then $\mathrm{M}_{p(\cdot)}\left(\Gamma_{E}\right)=0$.

Proof. For every $i \in \mathbb{N}$ we choose a function $u_{i} \in N^{1, p(\cdot)}(X)$ with weak upper gradient $h_{i}$ such that $u_{i}(x) \geqslant 1$ for every $x \in E$ and

$$
\int_{X}\left|u_{i}(x)\right|^{p(x)}+h_{i}(x)^{p(x)} d \mu(x) \leqslant 2^{-i}
$$

We define

$$
v_{k}=\sum_{i=1}^{k}\left|u_{i}\right|
$$

We find that $\rho_{k}=\sum_{i=1}^{k} h_{i}$ is a weak upper gradient of $v_{k}$. For every $l>m$ we find that

$$
\left\|v_{l}-v_{m}\right\|_{p(\cdot)} \leqslant \sum_{i=m+1}^{l}\left\|u_{i}\right\|_{p(\cdot)} \leqslant 2^{-m}
$$

and

$$
\left\|\rho_{l}-\rho_{m}\right\|_{p(\cdot)} \leqslant \sum_{i=m+1}^{l}\left\|h_{i}\right\|_{p(\cdot)} \leqslant 2^{-m}
$$

and therefore the sequences $\left(v_{k}\right)_{k=1}^{\infty}$ and $\left(\rho_{k}\right)_{k=1}^{\infty}$ are Cauchy sequences in the Banach space $L^{p(\cdot)}(X)$. So $\left(\rho_{k}\right)$ converges to a function $\rho$ in $L^{p(\cdot)}(X)$, which we may assume to be Borel. Since the sequence $\left(v_{k}(x)\right)$ is non-negative and increasing for every $x \in X$ the limit $v(x)=\lim _{k \rightarrow \infty} v_{k}(x)$ (possibly $+\infty$ ) exists for every $x \in X$ and $v \in L^{p(\cdot)}(X)$. For $x \in E$ we see that $v_{k}(x) \geqslant k$ for every $k$ and thus

$$
E \subset E_{\infty}=\left\{x \in X: \lim _{k \rightarrow \infty} v_{k}(x)=\infty\right\}
$$

Therefore it suffices to show that $\mathrm{M}_{p(\cdot)}\left(\Gamma_{E_{\infty}}\right)=0$.

Lemma 3.1 gives a subsequence of $\left(\rho_{k}\right)$, denoted again by $\left(\rho_{k}\right)$, such that there is an exceptional family $\Gamma_{1}$ and

$$
\lim _{k \rightarrow \infty} \int_{\gamma}\left|\rho_{k}-\rho\right| d s=0
$$


for every rectifiable curve $\gamma \notin \Gamma_{1}$. Let $\Gamma_{2}$ be the family of all curves $\gamma$ such that $\int_{\gamma} v d s=\infty$ and $\Gamma_{3}$ the family of curves $\gamma$ with $\int_{\gamma} \rho d s=\infty$. Since $v / i$ is admissible for $\Gamma_{2}$ and every $i=1,2, \ldots$ and since $v \in L^{p(\cdot)}\left(\mathbb{R}^{n}\right)$, we find that

$$
\mathrm{M}_{p(\cdot)}\left(\Gamma_{2}\right) \leqslant \int_{X}\left(\frac{v(x)}{i}\right)^{p(x)} d x \leqslant \frac{\|v\|_{p(\cdot)}}{i}
$$

for all $i \geqslant\|v\|_{p(\cdot)}$, by [21, (2.11)]. Therefore $\mathrm{M}_{p(\cdot)}\left(\Gamma_{2}\right)=0$ and similarly $\mathrm{M}_{p(\cdot)}\left(\Gamma_{3}\right)=0$. Let $\Gamma_{4, i}$ be the exceptional family of curves from the definition of $u_{i}$. By subadditivity we obtain that $\mathrm{M}_{p(\cdot)}\left(\Gamma_{4}\right)=\mathrm{M}_{p(\cdot)}\left(\bigcup \Gamma_{4, i}\right)=0$. This yields that $\mathrm{M}_{p(\cdot)}\left(\Gamma^{*}\right)=0$, where $\Gamma^{*}=\Gamma_{1} \cup \Gamma_{2} \cup \Gamma_{3} \cup \Gamma_{4}$.

To complete the proof we show that $\Gamma_{E_{\infty}} \subset \Gamma^{*}$. Suppose that $\gamma \notin \Gamma^{*}$. Since $\gamma \notin \Gamma_{2}$ there is $y \in|\gamma|$ with $v(y)<\infty$. For any point $x \in|\gamma|$ we find since $\gamma \notin \Gamma_{4}$ that

$$
\left|v_{i}(x)\right| \leqslant\left|v_{i}(y)\right|+\left|v_{i}(x)-v_{i}(y)\right| \leqslant\left|v_{i}(y)\right|+\int_{\gamma} \rho_{i} d s .
$$

Taking the limit as $i \rightarrow \infty$ in this inequality gives, using (3.3) and $\gamma \notin \Gamma_{1}$ for the inequality, that

$$
v(x)=\lim _{i \rightarrow \infty}\left|v_{i}(x)\right| \leqslant \lim _{i \rightarrow \infty}\left|v_{i}(y)\right|+\int_{\gamma} \rho d s .
$$

Since $\gamma \notin \Gamma_{3}$ and $v(y)<\infty$, the right-hand-side is finite. Hence $v(x)<\infty$ for all $x \in|\gamma|$, which implies that $\gamma \notin \Gamma_{E_{\infty}}$. Thus $\Gamma_{E_{\infty}} \subset \Gamma^{*}$, which completes the proof.

Theorem 3.4. If $p^{+}<\infty$, then $N^{1, p(\cdot)}(X)$ is a Banach space.

Proof. Let $u_{i}$ be a Cauchy sequence in $N^{1, p(\cdot)}(X)$. Passing to a subsequence if necessary we assume that

$$
\left\|u_{i+1}-u_{i}\right\|_{N^{1, p(\cdot)}(X)}<2^{-2 i} .
$$

Let $\rho_{i}^{\prime}$ be a weak upper gradient of $2^{i}\left|u_{i+1}-u_{i}\right|$ such that

$$
2^{i}\left\|u_{i+1}-u_{i}\right\|_{p(\cdot)}+\left\|\rho_{i}^{\prime}\right\|_{p(\cdot)}<2^{-i} .
$$

Let $E_{i}=\left\{x \in X:\left|u_{i+1}(x)-u_{i}(x)\right|>2^{-i}\right\}$. Then $2^{i}\left|u_{i+1}-u_{i}\right| \in N^{1, p(\cdot)}(X)$ and $2^{i}\left|u_{i+1}-u_{i}\right| \geqslant 1$ in $E_{i}$. Hence we obtain, using $2^{i}\left|u_{i+1}-u_{i}\right|$ as a test function for the capacity,

$$
c_{p(\cdot)}\left(E_{i}\right) \leqslant \varrho_{p(\cdot)}\left(2^{i}\left|u_{i+1}-u_{i}\right|\right)+\varrho_{p(\cdot)}\left(\rho_{i}^{\prime}\right) \leqslant 2^{i}\left\|u_{i+1}-u_{i}\right\|_{p(\cdot)}+\left\|\rho_{i}^{\prime}\right\|_{p(\cdot)} \leqslant 2^{-i} .
$$

Let $F_{j}=\bigcup_{i=j}^{\infty} E_{i}$ and $F=\cap_{j \in \mathbb{N}} F_{j}$. Then we get $c_{p(\cdot)}\left(F_{j}\right) \leqslant \sum_{i=j}^{\infty} c_{p(\cdot)}\left(E_{i}\right) \leqslant$ $2^{-j+1}$ and $c_{p(\cdot)}(F)=0$. For $x \in X \backslash F$ the sequence $u_{i}(x)$ is a Cauchy sequence in $\mathbb{R}$ and we set $u(x)=\lim _{i \rightarrow \infty} u_{i}(x)$. 
Next we show that $u$ has a weak upper gradient. Let $g_{1}$ be a weak $p(\cdot)$-integrable upper gradient of $u_{1}$ and let $g_{i+1}$ that a weak upper gradient of $u_{i+1}-u_{i}$ with $\left\|g_{i}\right\|_{p(\cdot)} \leqslant 2^{-2 i}$ for $i=1,2, \ldots$ Define $\rho_{i}=g_{1}+\ldots+g_{i}$ and note that $\rho_{i}$ is a weak upper gradient of $u_{i}$. Since $\left(\rho_{i}\right)$ is a Cauchy sequence it converges to a function $\rho$ in $L^{p(\cdot)}(X)$. Passing to a subsequence, if necessary, we obtain by Lemma 3.1 that

$$
\lim _{i \rightarrow \infty} \int_{\gamma} \rho_{i} d s=\int_{\gamma} \rho d s
$$

except for $\gamma$ in a family of curves $\Gamma$ of zero $p(\cdot)$-modulus. By Lemma 3.2, the modulus of $\Gamma_{F}$ is zero. Hence we obtain for every rectifiable $\gamma \notin \Gamma \cup \Gamma_{F}$ joining $x, y \in X$ that

$$
|u(x)-u(y)|=\lim _{i \rightarrow \infty}\left|u_{i}(x)-u_{i}(y)\right| \leqslant \lim _{i \rightarrow \infty} \int_{\gamma} \rho_{i} d s=\int_{\gamma} \rho d s .
$$

The first equality follows since $x, y \notin F$, as they lie on a curve not in $\Gamma_{F}$. Thus we have shown that $\rho$ is a weak upper gradient of $u$.

Now we have only to prove that $u_{i} \rightarrow u$ in $L^{p(\cdot)}(X)$. For every $k \in \mathbb{N}$ we have

$$
\left\|u-u_{k}\right\|_{p(\cdot)} \leqslant \sum_{i=k}^{\infty}\left\|u_{i+1}-u_{i}\right\|_{p(\cdot)} \leqslant \sum_{i=k}^{\infty} 2^{-2 i} \leqslant 4^{1-k}
$$

This completes the proof of Theorem 3.4.

3.2. Density of Lipschitz continuous functions. Next we study when Lipschitz functions are dense in the Newtonian space. For this result we need to assume that the Hardy-Littlewood maximal operator is locally bounded from $L^{p(\cdot)}(X) \rightarrow L^{p(\cdot)}(X)$. For a summary of what is known about this, see Section 2.3.

We say that $X$ supports a $(1,1)$-Poincaré inequality if there exists a constant $C>0$ such that for all open balls $B$ in $X$ and all pairs of functions $u$ and $\rho$ defined on $B$ the inequality

$$
f_{B}\left|u-u_{B}\right| d \mu \leqslant C \operatorname{diam}(B) f_{B} \rho d \mu
$$

holds whenever $\rho$ is an upper gradient of $u$ on $B$ and $u$ in integrable on $B$ the inequality. The proof of the next theorem follows that of [29, Theorem 4.1].

Theorem 3.5. Let $X$ be a doubling space that supports a $(1,1)$-Poincaré inequality. Assume that $p^{+}<\infty$ and the Hardy-Littlewood maximal operator is bounded from $L^{p(\cdot)}(X) \rightarrow L^{p(\cdot)}(X)$. Then Lipschitz continuous functions are dense in $N^{1, p(\cdot)}(X)$.

Proof. It is easy to see by a truncation argument that bounded functions are dense in $N^{1, p(\cdot)}(X)$ (e.g. [29, Lemma 4.3]). Hence it suffices to consider the case 
of bounded $u \in N^{1, p(\cdot)}(X)$, say $|u(x)|<u_{0}$. Let $\rho \in L^{p(\cdot)}\left(\mathbb{R}^{n}\right)$ be a weak upper gradient of $u$. We set

$$
E_{\lambda}=\{x \in X: \mathcal{M} \rho(x)>\lambda\} .
$$

Note that $E_{\lambda}$ is open since $\mathcal{M}$ is lower semi-continuous. If $x \in X \backslash E_{\lambda}$, then for all $r>0$ and for balls $B=B(x, r)$ we have

$$
f_{B}\left|u-u_{B}\right| d \mu \leqslant \operatorname{cr} f_{B} \rho d \mu \leqslant \operatorname{cr} \mathcal{M} \rho(x) \leqslant c r \lambda .
$$

Hence for $s \in\left[\frac{r}{2}, r\right]$ and $x \in X \backslash E_{\lambda}$ the doubling property implies that

$$
\begin{aligned}
\left|u_{B(x, s)}-u_{B(x, r)}\right| & \leqslant f_{B(x, s)}\left|u-u_{B(x, r)}\right| d \mu \\
& \leqslant \frac{\mu(B(x, r))}{\mu(B(x, s))} f_{B(x, r)}\left|u-u_{B(x, r)}\right| d \mu \\
& \leqslant c r \lambda .
\end{aligned}
$$

Using this inequality for $r=r_{0}, r_{0} / 2, r_{0} / 4, \ldots$ we find that

$$
\begin{aligned}
\left|u_{B(x, s)}-u_{B(x, r)}\right| & \leqslant\left|u_{B(x, s)}-u_{B\left(x, 2^{-i} r\right)}\right|+\sum_{j=1}^{i}\left|u_{B\left(x, 2^{-j} r\right)}-u_{B\left(x, 2^{1-j} r\right)}\right| \\
& \leqslant\left(2^{-i} r+2^{1-i} r+\ldots+r\right) \lambda \leqslant c r \lambda,
\end{aligned}
$$

where $i$ is the largest integer for which $s \leqslant 2^{-i} r$. Hence any sequence $\left(u_{B\left(x, r_{i}\right)}\right)$, $r_{i} \rightarrow 0$, is a Cauchy sequence in $\mathbb{R}$. Therefore on $X \backslash E_{\lambda}$ we can define

$$
u_{\lambda}(x)=\lim _{r \rightarrow 0} u_{B(x, r)} .
$$

Since $\mu$-almost every point is a Lebesgue point for every function in $L_{l o c}^{1}(X)$ we note that $u(x)=u_{\lambda}(x)$ for $\mu$-almost every $x \in X \backslash E_{\lambda}$. For $x, y \in X$ we define a chain of balls $\left(B_{i}\right)_{i \in \mathbb{Z} \backslash\{0\}}$ by setting

$$
B_{1}=B(x, d(x, y)) \quad \text { and } \quad B_{-1}=B(y, d(x, y))
$$

and inductively

$$
B_{i}=\frac{1}{2} B_{i-1}(\text { for } i>1) \quad \text { and } \quad B_{-i}=\frac{1}{2} B_{-i+1}(\text { for } i<-1) .
$$

We calculate by the doubling property and the $(1,1)$-Poincaré inequality that

$$
\begin{aligned}
\mu\left(B_{1}\right)\left|u_{B_{1}}-u_{B_{-1}}\right| & \leqslant \mu\left(B_{1}\right)\left|u_{B_{1}}-u_{2 B_{1}}\right|+\mu\left(B_{1}\right)\left|u_{2 B_{1}}-u_{B_{-1}}\right| \\
& \leqslant \int_{B_{1}}\left|u-u_{2 B_{1}}\right| d \mu+\frac{\mu\left(B_{1}\right)}{\mu\left(B_{-1}\right)} \int_{B_{-1}}\left|u-u_{2 B_{1}}\right| d \mu \\
& \leqslant \int_{2 B_{1}}\left|u-u_{2 B_{1}}\right| d \mu+\frac{\mu\left(2 B_{-1}\right)}{\mu\left(B_{-1}\right)} \int_{2 B_{1}}\left|u-u_{2 B_{1}}\right| d \mu \\
& \leqslant C \operatorname{diam}\left(B_{1}\right) \int_{2 B_{1}} \rho d \mu .
\end{aligned}
$$


Dividing by $\mu\left(B_{1}\right)$ and using the doubling property we get

$$
\left|u_{B_{1}}-u_{B_{-1}}\right| \leqslant c d(x, y) \mathcal{M} \rho(x) \leqslant c d(x, y) \lambda .
$$

If $x, y \in X \backslash E_{\lambda}$, then they are Lebesgue points also of $u_{\lambda}$ and hence

$$
\begin{aligned}
\left|u_{\lambda}(x)-u_{\lambda}(y)\right| & \leqslant \sum_{i=1}^{\infty}\left|u_{B_{i+1}}-u_{B_{i}}\right|+\left|u_{B_{1}}-u_{B_{-1}}\right|+\sum_{i=1}^{\infty}\left|u_{B_{-i-1}}-u_{B_{-i}}\right| \\
& \leqslant c \lambda d(x, y) .
\end{aligned}
$$

Hence $u_{\lambda}$ is $c \lambda$-Lipschitz in $X \backslash E_{\lambda}$. We extend $u_{\lambda}$ as a Lipschitz function to all of $X$ by McShane extension, [22], setting

$$
u_{\lambda}(x)=\inf _{y \in X \backslash E_{\lambda}}\left\{u_{\lambda}(y)+c \lambda d(x, y)\right\} .
$$

We may assume that the extension is bounded by $u_{0}$. This can be done by truncation. Then we easily see that $u_{\lambda} \rightarrow u$ in $L^{p(\cdot)}(X)$.

Non-zero values of $u-u_{\lambda}$ are obtained only at points in $E_{\lambda}$ and on a set $L$ whose measure is zero. Since $E_{\lambda}$ is open and $u-u_{\lambda}$ is zero $\mu$-almost everywhere in the complement of $E_{\lambda}$, we may assume by [29, Lemma 4.3] that the upper gradient of $u-u_{\lambda}$ is zero in $X \backslash E_{\lambda}$. Notice that $\lambda \chi_{E_{\lambda}} \in L^{p(\cdot)}(X)$ by the definition of $\lambda$ since $\mathcal{M} u$ is in $L^{p(\cdot)}(X)$. Therefore we find that the function $(c \lambda+\rho) \chi_{E_{\lambda}}$ is a weak upper gradient of $u-u_{\lambda}$. Hence $u-u_{\lambda}$ is in $N^{1, p(\cdot)}(X)$ and therefore so is $u_{\lambda}$. We obtain

$$
\begin{aligned}
\int_{X}\left|(c \lambda+\rho(x)) \chi_{E_{\lambda}}(x)\right|^{p(x)} d \mu(x) & =\int_{E_{\lambda}}|c \lambda+\rho(x)|^{p(x)} d \mu(x) \\
& \leqslant C \int_{E_{\lambda}}|\rho(x)|^{p(x)}+|\lambda|^{p(x)} d \mu(x) \\
& \leqslant C \int_{E_{\lambda}}|u(x)|^{p(x)}+[\mathcal{N} \rho(x)]^{p(x)} d \mu(x) .
\end{aligned}
$$

Since both $\rho$ and $\mathcal{M} \rho$ belong to $L^{p(\cdot)}(X)$, the right hand side converges to zero as $\lambda \rightarrow \infty$. Hence the sequence $u_{\lambda}$ converges to $u$ in $N^{1, p(\cdot)}(X)$.

Remark 3.6. It is easy to see that the approximating functions constructed in the previous theorem has the additional property of being non-negative whenever the function itself is.

\section{Equivalence of function spaces}

In this section we study when Hajłasz, Newtonian and classical Sobolev spaces agree. We will see that, roughly speaking, Hajłasz space agrees with Sobolev space 
if the maximal operator is bounded, whereas the Newtonian space agrees with Sobolev space if differentiable functions are dense. This reflects the fact that the boundedness of the maximal function is somehow built into the definition of Hajłasz space, as the density of differentiable functions is into Newtonian space. For what is known about when these conditions hold, see Sections 2.3 and 2.4.

Throughout this section we restrict our attention to exponents $p: X \rightarrow$ $(1, \infty)$ not taking the value 1 . We say that a $p(\cdot)$-integrable function $u$ belongs to Hajłasz space $M^{1, p(\cdot)}(X, d, \mu)=M^{1, p(\cdot)}(X)$ if there exists a non-negative $g \in$ $L^{p(\cdot)}(X)$ such that

$$
|u(x)-u(y)| \leqslant d(x, y)(g(x)+g(y))
$$

for $\mu$-almost every $x, y \in X$. The function $g$ is called a Hajtasz gradient of $u$. We equip $M^{1, p(\cdot)}(X)$ with the norm

$$
\|u\|_{M^{1, p(\cdot)(X)}}=\|u\|_{p(\cdot)}+\inf \|g\|_{p(\cdot)},
$$

where the infimum is taken over all Hajłasz gradients of $u$. Following the arguments in [12] it is easy to prove that $M^{1, p(\cdot)}(X)$ is a Banach space and Lipschitz continuous functions are dense, provided that $p^{+}<\infty$.

Proposition 4.1. We have $M^{1, p(\cdot)}\left(\mathbb{R}^{n}\right) \subset W^{1, p(\cdot)}\left(\mathbb{R}^{n}\right)$. If the maximal operator is bounded from $L^{p(\cdot)}\left(\mathbb{R}^{n}\right)$ to itself, then $M^{1, p(\cdot)}\left(\mathbb{R}^{n}\right)=W^{1, p(\cdot)}\left(\mathbb{R}^{n}\right)$.

Proof. Fix first $u \in M^{1, p(\cdot)}\left(\mathbb{R}^{n}\right)$ and let $g \in L^{p(\cdot)}\left(\mathbb{R}^{n}\right)$ be a nonnegative function such that

$$
|u(x)-u(y)| \leqslant|x-y|(g(x)+g(y))
$$

for almost every $x, y \in X$. We know that $g \in L^{1}(B)$ in every ball $B$ and hence by [13, Proposition 1] (or [18, Remark 5.13]) $\nabla u$ exists and satisfies $|\nabla u| \leqslant C(n) g$ almost everywhere. Thus we obtain that $|\nabla u| \in L^{p(\cdot)}\left(\mathbb{R}^{n}\right)$, and so $u \in W^{1, p(\cdot)}\left(\mathbb{R}^{n}\right)$, since $u \in L^{p(\cdot)}\left(\mathbb{R}^{n}\right)$ by definition.

To prove the second claim we fix $u \in W^{1, p(\cdot)}\left(\mathbb{R}^{n}\right)$ and assume that $\mathcal{M}$ is bounded. We find as in [12, Chapter 2] that

$$
|u(x)-u(y)| \leqslant|x-y|(\mathcal{M}|\nabla u|(x)+\mathcal{M}|\nabla u|(y)) .
$$

for almost every $x, y \in \mathbb{R}^{n}$. Since the maximal operator $\mathcal{M}$ is bounded in $L^{p(\cdot)}\left(\mathbb{R}^{n}\right)$, we find that $\mathcal{M}|\nabla u|$ is a Hajłasz gradient of $u$ in $L^{p(\cdot)}\left(\mathbb{R}^{n}\right)$, and so $u \in M^{1, p(\cdot)}\left(\mathbb{R}^{n}\right)$.

We give two alternative characterizations of the Hajłasz space. For this purpose we introduce a fractional sharp maximal operator. For every locally integrable function $u$ we define

$$
u^{\#}(x)=\sup _{r>0} \frac{1}{r} f_{B(x, r)}\left|u(x)-u_{B(x, r)}\right| d \mu(x) .
$$

In the variable exponent setting the sharp maximal operator has been studied by Diening and Růžička $[6,7]$. The following theorem is a generalization of [14, Theorem 3.4], and the proof given in that paper also works in our case. 
Theorem 4.2. If the Hardy-Littlewood maximal operator is bounded from $L^{p(\cdot)}(X)$ to itself, then the following three statements are equivalent:

(i) $u \in M^{1, p(\cdot)}(X)$;

(ii) $u \in L^{p(\cdot)}(X)$ and there exists a non-negative $g \in L^{p(\cdot)}(X)$ such that

$$
f_{B(x, r)}\left|u-u_{B(x, r)}\right| d \mu \leqslant c r f_{B(x, r)} g d \mu
$$

holds for every $x \in X$ and $r>0$;

(iii) $u \in L^{p(\cdot)}(X)$ and $u^{\#} \in L^{p(\cdot)}(X)$.

Moreover, we have that

$$
\begin{aligned}
\|u\|_{M^{1, p(\cdot)}(X)} & \approx\|u\|_{p(\cdot)}+\inf \left\{\|g\|_{p(\cdot)}: g \text { as in (ii) }\right\} \\
& \approx\|u\|_{p(\cdot)}+\left\|u^{\#}\right\|_{p(\cdot)} .
\end{aligned}
$$

Theorem 4.3. Let $\Omega \subset \mathbb{R}^{n}$ be an open set. We have $N^{1, p(\cdot)}(\Omega) \subset W^{1, p(\cdot)}(\Omega)$. If $1<p^{-} \leqslant p^{+}<\infty$ and $C^{1}(\Omega)$ is dense in $W^{1, p(\cdot)}(\Omega)$, then $N^{1, p(\cdot)}(\Omega)=$ $W^{1, p(\cdot)}(\Omega)$.

Proof. Let $u \in N^{1, p(\cdot)}\left(\mathbb{R}^{n}\right)$. Then $u$ is absolutely continuous on every curve except a family of zero $p(\cdot)$-modulus, and hence $u$ has classical derivatives almost everywhere. Denote by $\rho$ a weak upper gradient of $u$.

Let $Q=(-r, r)^{n}$ for $r>0$. We have that

$$
\begin{aligned}
& \int_{(-r, r)^{n-1}} \int_{(-r, r)} \rho(t, y) d t d H^{n-1}(y) \\
& \leqslant \int_{Q} \rho(x) d x \leqslant(1+|Q|) \int_{Q} \rho(x)^{p(x)} d x<\infty,
\end{aligned}
$$

which means that $\int_{(-r, r)} \rho(t, x) d t<\infty$ except in a set $E_{k}$ with $m_{n-1}\left(E_{k}\right)=0$. For $y \notin E_{k} H^{1}$-almost every point in $((-r, y),(r, y))$ is a Lebesgue point. Thus for almost every point in $Q$ we have

$$
\lim _{t \rightarrow 0} f_{\left[y, y+t e_{1}\right]} \rho d s=\rho(y),
$$

where $[z, w]$ denotes the line segment joining $z$ and $w$. On the other hand we have

$$
\frac{1}{|t|}\left|u(y)-u\left(y+t e_{1}\right)\right| \leqslant f_{\left[y, y+t e_{1}\right]} \rho d s,
$$

Since $u$ is differentiable almost everywhere, this implies that $\left|\partial_{1} u(x)\right| \leqslant \rho(x)$ almost everywhere in $Q$. Letting $r \rightarrow \infty$ and using the subadditivity we derive the same claim in all of $\mathbb{R}^{n}$. Finally the same argument applies in directions $e_{2}, \ldots, e_{n}$ as well, so we get $|\nabla u(x)| \leqslant \sqrt{n} \rho(x)$ for almost every $x$. Therefore $|\nabla u| \in L^{p(\cdot)}\left(\mathbb{R}^{n}\right)$ and so $u \in W^{1, p(\cdot)}\left(\mathbb{R}^{n}\right)$. 
To prove the inclusion $W^{1, p(\cdot)}(\Omega) \subset N^{1, p(\cdot)}(\Omega)$ we need to show that every function in $W^{1, p(\cdot)}(\Omega)$ has a weak upper gradient. But it follows as in [16, Theorem 4.2] that the distributional gradient is an upper gradient in $\mathbb{R}^{n}$. From this it follows that the same claim holds in $\Omega \subset \mathbb{R}^{n}$ using the argument of [16, Theorem 4.6].

Finally we prove relations between Hajłasz and Newtonian spaces. The following theorem is a generalization of [29, Lemma 4.8].

Theorem 4.4. Let $p^{+}<\infty$. Then $M^{1, p(\cdot)}(X) \subset N^{1, p(\cdot)}(X)$ and $\|u\|_{N^{1, p(\cdot)}} \leqslant$

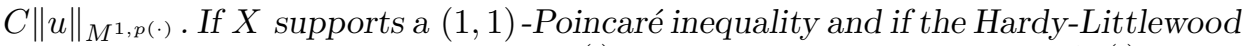
maximal operator is bounded from $L^{p(\cdot)}(X)$ to itself, then we have $M^{1, p(\cdot)}(X)=$ $N^{1, p(\cdot)}(X)$ and the norms are comparable.

Proof. Let $u \in M^{1, p(\cdot)}(X)$. If $u$ is continuous, we find as in [29, Lemma 4.7] that $4 g$ is an upper gradient for $u$, where $g$ is the Hajłasz gradient of $u$. Since continuous functions are dense in $M^{1, p(\cdot)}(X)$, we can approximate $u \in M^{1, p(\cdot)}(X)$ with continuous functions $u_{i}$. Since $u_{i} \rightarrow u, g_{i} \rightarrow g_{u}$, and $N^{1, p(\cdot)}(X)$ is a Banach space by Theorem 3.4, we find that $4 g$ is an upper gradient of $u$.

Next let $u \in N^{1, p(\cdot)}(X)$, and let $\rho$ be a weak upper gradient of $u$. Since by the definition $\rho \in L^{p(\cdot)}(X), \rho$ is non-negative and $X$ supports $(1,1)$-Poincaré inequality, the inclusion $M^{1, p(\cdot)}(X) \supset N^{1, p(\cdot)}(X)$ follows by Theorem 4.2. This completes the proof.

Acknowledgement. The authors wish to thank Ilkka Holopainen and Olli Martio for comments on this manuscript.

\section{References}

[1] A. Björn, J. Björn and N. Shanmugalingam, The Dirichlet problem for p-harmonic functions on metric spaces, J. Reine Angew. Math. 556 (2003), 173-203.

[2] J. Cheeger, Differentiability of Lipschitz functions on metric measure spaces, Geom. Funct. Anal. 9 (1999), 428-517.

[3] D. Cruz-Uribe, A. Fiorenze and C. J. Neugebauer, The maximal operator on variable $L^{p}$ spaces, Ann. Acad. Sci. Fenn. Math. 28 (2003), 223-238; 29 (2004), 247-249.

[4] L. Diening, Maximal operator on generalized Lebesgue spaces $L^{p(\cdot)}$, Math. Inequal. Appl. 7 (2004), no. 2, 245-253.

[5] L. Diening, Maximal Function on Musielak Orlicz Spaces and Generalized Lebesgue Spaces, Bull. Sci. Math. 129 (2005), no. 8, 675-700.

[6] L. Diening and M. Růžička, Calderón-Zygmund operators on generalized Lebesgue spaces $L^{p(\cdot)}$ and problems related to fluid dynamics, J. Reine Angew. Math. 563 (2003), 197-220.

[7] L. Diening and M. Růžička, Integral operators on the halfspace in generalized Lebesgue spaces $L^{p(\cdot)}$, (I) J. Math. Anal. Appl. 298 (2004), no. 2, 559-571; (II) J. Math. Anal. Appl. 298 (2004), no. 2, 572-588. 
[8] D. E. Edmunds and J. Rákosník, Density of smooth functions in $W^{k, p(x)}(\Omega)$, Proc. Roy. Soc. London Ser. A 437 (1992), 229-236.

[9] X. Fan and D. Zhao, On the spaces $L^{p(x)}(\Omega)$ and $W^{m, p(x)}(\Omega)$, J. Math. Anal. Appl. 263 (2001), 424-446.

[10] T. Futamura, Y. Mizuta and T. Shimomura, Sobolev embeddings for variable exponent Riesz potentials on metric spaces, Ann. Acad. Sci. Fenn. Math. 31 (2006), no. 2, 495-522.

[11] B. Fuglede, Extremal length and functional completion, Acta Math. 98 (1957), 171-218.

[12] P. Hajtasz, Sobolev spaces on arbitrary metric spaces, Potential Anal. 5 (1996), 403-415.

[13] P. Hajłasz, Geometric approach to Sobolev spaces and badly degenerated elliptic equations, in Nonlinear Analysis and Applications (Warzawa, 1994; N. Kenmochi et all. (eds.)) GAGUTO Internat. Ser. Math. Sci. Appl. 7, Gakkotosho, Tokyo, 1996, 141-168.

[14] P. Hajłasz and J. Kinnunen, Hölder quasicontinuity of Sobolev functions on metric spaces, Rev. Mat. Iberoamericana 14 (1998), no. 3, 601-622.

[15] P. Harjulehto, P. Hästö, M. Koskenoja and S. Varonen, Sobolev capacity on the space $W^{1, p(\cdot)}\left(\mathbb{R}^{n}\right)$, J. Funct. Spaces Appl. 1 (2003), no. 1, 17-33.

[16] P. Harjulehto, P. Hästö and O. Martio, Fuglede's theorem in variable exponent Sobolev space, Collect. Math. 55, (2004), no. 3, 315-324.

[17] P. Harjulehto, P. Hästö and M. Pere, Variable exponent Lebesgue spaces on metric spaces, the Hardy-Littlewood maximal operator, Real Anal. Exchange $30(2004 / 05)$, no. 1, 87-104.

[18] J. Heinonen, Lectures on analysis on metric spaces, Springer-Verlag, New York, 2001.

[19] J. Kinnunen and O. Martio, Potential theory of quasiminimizers, Ann. Acad. Sci. Fenn. Math. 28 (2003), no. 2, 459-490.

[20] V. Kokilasvili and S. Samko, Maximal and fractional operators in weighted $L^{p(x)}$ spaces, Rev. Mat. Iberoamericana 20 (2004), no. 2, 493-515.

[21] O. Kováčik and J. Rákosník, On spaces $L^{p(x)}$ and $W^{1, p(x)}$, Czechoslovak Math. J. 41(116) (1991), 592-618.

[22] E. J. McShane, Extension of range of functions, Bull. Am. Math. Soc. 40 (1934), 837-842.

[23] Y. Mizuta and T. Shimomura, Continuity of Sobolev functions of variable exponent on metric spaces, Proc. Japan Acad. Ser. A Math. Sci. 80 (2004), no. 6, 96-99.

[24] J. Musielak, Orlicz Spaces and Modular Spaces, Springer-Verlag, Berlin, 1985.

[25] A. Nekvinda, Hardy-Littlewood maximal operator on $L^{p(x)}\left(\mathbb{R}^{n}\right)$, Math. Inequal. Appl. 7 (2004), no. 2, 255-265.

[26] A. Nekvinda, A note on maximal operator on $l^{p_{n}}$ and $L^{p(x)}\left(\mathbb{R}^{n}\right)$, preprint (2004).

[27] L. Pick and M. Růžička, An example of a space $L^{p(x)}$ on which the Hardy-Littlewood maximal operator is not bounded, Expo. Math. 19 (2001), 369-371. 
[28] S. Samko, Denseness of $C_{0}^{\infty}\left(\mathbb{R}^{n}\right)$ in the generalized Sobolev spaces $W^{m, p(x)}\left(\mathbb{R}^{n}\right)$, pp. 333-342 in Direct and inverse problems of mathematical physics (Newark, DE, 1997), Int. Soc. Anal. Appl. Comput. 5, Kluwer Acad. Publ., Dordrecht, 2000.

[29] N. Shanmugalingam, Newtonian spaces, An extension of Sobolev spaces to metric measure space, Rev. Mat. Iberoamericana 16 (2000), no. 2, 243-279.

[30] I. Sharapudinov, On the topology of the space $L^{p(t)}([0 ; 1])$, Math. Notes 26 (1979), no. 3-4, 796-806. [translation of Mat. Zametki 26 (1978), no. 4, 613-632.]

Addresses: Department of Mathematics and Statistics, P.O. Box 68, FI-00014 University of Helsinki, Finland

Research group home-page: http://www.math.helsinki.fi/analysis/varsobgroup/

E-mail: petteri.harjulehto@helsinki.fi; peter.hasto@helsinki.fi

Received: 21 December 2004; revised: 15 April 2005 